\title{
Lesão por pressão associada à contenção mecânica: estudo transversal
}

\section{Pressure injury associated with mechanical restraint: a cross-sectional study}

Lesión por presión asociada a la contención mecánica: estudio transveral

Livia Maria da Silva Souza', Rosimere Ferreira Santana ${ }^{2, *}$, Marcia Verissimo de Souza ${ }^{1}$, Simone Martins Rembold ${ }^{2}$, Arianna Kassiadou Menezes ${ }^{3}$

ORCID IDS

Souza LMS (D) https://orcid.org/0000-0002-5225-2045

Santana RF (D) https://orcid.org/0000-0002-4593-3715

Souza MV (D) https://orcid.org/0000-0001-9627-9824

Rembold SM (D) https://orcid.org/0000-0003-1424-747X

Menezes AK (iD https://orcid.org/0000-0002-9159-3002

\section{COMO CITAR}

Souza LMS, Santana RF, Souza MV, Rembold SM, Menezes AK. Lesão por pressão associada à contenção mecânica: estudo transversal. ESTIMA, Braz. J. Enterostomal Ther., 17: e0919. https://doi. org/10.30886/estima.v17.703_PT

\begin{abstract}
RESUMO
Objetivo: Verificar associação da prática de contenção mecânica com lesão por pressão (LP) em pacientes hospitalizados. Método: Estudo transversal realizado com 111 pacientes de unidades de clínica médica, cirúrgica e de terapia intensiva em um hospital público do estado do Rio de Janeiro, Brasil. Para analisar a associação entre as variáveis, adotou-se a razão de chances [odds ratio (OR)]. Resultados: Encontraram-se 57 pacientes em contenção mecânica (51,4\%). No grupo de pacientes contidos, a ocorrência de LP foi estimada em 43,9\% e, no grupo de não contidos, em 5,6\%. A chance de o paciente contido apresentar LP foi 13 vezes maior do que em pacientes não contidos. A localização da lesão foi mais frequentemente na região sacra, classificada em estágio 2 (21,1\%) e estágio 3 (12,3\%), seguida de trocânter (15,8\%) e calcâneo (10,5\%). Conclusão: Recomenda-se a adoção de práticas de enfermagem de supressão ou redução do tempo no uso da contenção mecânica, adotando medidas educativas e de prevenção da LP.
\end{abstract}

DESCRITORES: Estomaterapia; Úlcera por pressão; Restrição física.

\footnotetext{
1. Universidade Federal Fluminense - Escola de Enfermagem Aurora de Afonso Costa - Niterói/RJ - Brasil.

2.Universidade Federal Fluminense - Escola de Enfermagem Aurora de Afonso Costa - Departamento de Enfermagem Médico-

Cirúrgica - Niterói/RJ - Brasil.

3.Universidade Federal Fluminense - Escola de Enfermagem Aurora de Afonso Costa - Programa de Pós-Graduação em Ciências do Cuidado em Saúde - Niterói/RJ - Brasil.

*Autor correspondente: rosifesa@gmail.com

Recebido: 25 Jan 2019 | Aceito: 10 Maio 2019
} 
ABSTRACT

Objective: To verify the association of the practice of mechanical restraint with pressure injury (PI) in hospitalized patients. Method: A cross-sectional study of 111 patients from medical, surgical and intensive care units at a public hospital in the state of Rio de Janeiro, Brazil. To analyze the association between variables, the [odds ratio (OR)] was adopted. Results: It were found 57 patients in mechanical restraint (51.4\%). In the group of patients contained, the occurrence of PI was estimated in $43.9 \%$ and in the group of patients not contained in 5.6\%. The chance of the contained patient to present PI was 13 times higher than in patients not contained. The location of the injury was more frequently in the sacral region, classified as stage 2 (21.1\%) and stage 3 (12.3\%), followed by trochanter (15.8\%) and calcaneus (10.5\%). Conclusion: It is recommended the adoption of nursing practices of suppression or reduction of the time in the use of mechanical restraint, adopting educational measures and prevention of $\mathrm{PI}$.

DESCRIPTORS: Stomatherapy; Pressure ulcer; Physical restraint.

\section{RESUMEN}

Objetivo: Verificar la asociación de la práctica de contención mecánica con lesión por presión (LP) en pacientes hospitalizados. Método: Estudio transversal realizado con 111 pacientes de unidades de clínica médica, quirúrgica y de terapia intensiva en un hospital público del estado de Río de Janeiro, Brasil. Para analizar la asociación entre las variables, se adoptó la razón de posibilidades [odds ratio (OR)]. Resultados: Se encontraron 57 pacientes en contención mecánica (51,4\%). En el grupo de pacientes contenidos, la ocurrencia de LP fue estimada en el 43,9\% y, en el grupo de no contenidos, en el 5,6\%. La posibilidad de que el paciente contenía presentar LP fue 13 veces mayor que en pacientes no contenidos. La localización de la lesión fue más frecuentemente en la región sacra, clasificada en etapa $2(21,1 \%)$ y etapa $3(12,3 \%)$, seguida de trocánter $(15,8 \%)$ y calcáneo $(10,5 \%)$. Conclusión: Se recomienda la adopción de prácticas de enfermería de supresión o reducción del tiempo en el uso de la contención mecánica, adoptando medidas educativas y de prevención de la LP.

DESCRIPTORES: Estomaterapia; Úlcera por presíon; Restricción física.

\section{INTRODUÇÃO}

A contenção mecânica é frequentemente utilizada em hospitais com a finalidade de evitar quedas, controlar pacientes com agitação psicomotora e evitar a interrupção de um tratamento. Entretanto, seu uso é questionado por restringir a autonomia e a liberdade do paciente, além de estar relacionado a potenciais eventos adversos ${ }^{1,2}$.

Considera-se contenção mecânica o uso de dispositivos que restringem o movimento do indivíduo para uma posição de sua escolha e/ou o acesso ao seu próprio corpo ${ }^{5}$. No ambiente hospitalar, os dispositivos mais utilizados para contenção são faixas aplicadas nos pulsos, cotovelos, tornozelos e abdome ${ }^{1,3}$. As grades laterais do leito são consideradas, em alguns estudos, equipamentos de segurança na prevenção de quedas².

O uso inapropriado da contenção mecânica, amplamente estudado em instituições de longa permanência para idosos (ILPIs), pode causar danos que variam de intensidade. São citados desde comprometimento das habilidades cognitivas, atrofia muscular ou piora de atrofia existente, lesão por pressão (LP), incontinência urinária e fecal, contraturas, lesões, pneumonia, trombose venosa profunda, até danos fatais por asfixia ${ }^{2,4,5,22}$.
A população idosa tem sido mais exposta à contenção no ambiente hospitalar, tendo como contribuintes a dificuldade de mobilidade, o risco de queda, a dependência e a polifarmácia ${ }^{3}$. Informações sobre eventos adversos associados à contenção mecânica em hospitais gerais ainda são exíguas. Estudos conduzidos em hospitais relatam LP e infecção do trato urinário relacionadas a cateter como eventos provenientes do uso de contenção $0^{3,6}$. Em idosos, as complicações resultam em maior morbidade, mortalidade e custos ${ }^{6}$.

O estudo conduzido em um hospital de Israel, com 2.163 pacientes internados em clínicas médicas e cirúrgicas e em unidades de terapia intensiva, relatou a presença de LP como fator preditor para uso de contenção. Nesse estudo, as grades laterais dos leitos não foram consideradas dispositivos de contenção ${ }^{3}$.

As LPs se constituem em um problema complexo, multifatorial, resultando em elevados custos em nível individual, familiar e socioeconômico ${ }^{6,8,11}$. O surgimento da LP causa vários transtornos físicos e emocionais ao paciente, como desconforto, dor, estresse, aumento de risco de complicações, hospitalização prolongada, influência na morbidade e mortalidade ${ }^{8,12}$. 
A LP é considerada problema comum, sobretudo em idosos e em pessoas imobilizadas, e ocorre em aproximadamente $10 \%$ dos pacientes hospitalizados ${ }^{13,14}$. Evidencia-se o aumento em sua prevalência nos últimos anos devido a maior expectativa de vida ${ }^{8}$. A prevalência das LPs aumenta com a idade, ocorrendo em 50 a $70 \%$ dos pacientes com mais de 75 anos $^{9}$. Apesar de ser frequente, a ocorrência dessa lesão é presumidamente evitável em pelo menos $95 \%$ dos pacientes ${ }^{10,15}$. Nesse sentido, a Portaria No 529 de 2013 do Ministério da Saúde instituiu o Programa Nacional de Segurança do Paciente, com objetivo de reduzir, a um mínimo aceitável, o risco de dano associado ao cuidado de saúde. O programa explicita que a LP é considerada evento adverso, já que resulta em dano para o paciente, e sua ocorrência é de notificação compulsória mensal23.

Fatores intrínsecos e extrínsecos podem afetar as condições da pele e tecidos moles, reduzindo a resistência à pressão e cisalhamento, dentre os quais idade avançada, perda de sensibilidade, alteração do nível de consciência, dependência no autocuidado, hospitalização prolongada, microclima, redução da mobilidade ou imobilidade, diabetes, hipertensão, baixa pressão arterial diastólica, incontinência urinária ou anal, presença de espasmos musculares, estado nutricional deficitário, anemia, extremos do índice de massa corporal, deficiência imunológica e tabagismo ${ }^{8,11}$. O uso de medicamentos também pode contribuir para esse tipo de lesão, tais como corticosteróides ${ }^{8}$, antibióticos, anti-inflamatórios ${ }^{13} \mathrm{e}$ anticoagulantes ${ }^{14}$. Outros fatores que podem ser inapropriados na prática do cuidado merecem ser destacados, como o uso de dispositivos médicos, reposicionamento e/ou superfície de apoio, higiene e necessidade de proteção da pele seca, uso de hidratante ${ }^{16}$, uso de fralda e também de contenção mecânica ${ }^{11}$.

Portanto, esse estudo tem como objetivo verificar a associação da prática de contenção mecânica com a LP em pacientes hospitalizados.

\section{MÉTODOS}

Estudo observacional, transversal, realizado em um hospital público localizado em um município da mesorregião da Baixada Litorânea, no estado do Rio de Janeiro. O hospital realiza atendimento ambulatorial e hospitalar de média e alta complexidade e tem 70 leitos.

De acordo com dados do Departamento de Informática do Sistema Único de Saúde (Datasus), o hospital registrou 489 internações nos setores de internação de adultos no período de janeiro a agosto de 2016. Sendo assim, a população de interesse foi de 489 pacientes. Para consecução dos resultados de interesse, pela impossibilidade de se entrevistar toda a população, retirou-se uma amostra desse universo. $\mathrm{O}$ tamanho mínimo da amostra para este estudo foi de 106 pacientes contidos e não contidos.

Os critérios de inclusão foram adultos e idosos internados nos setores de clínica médica, clínica cirúrgica e unidade de terapia intensiva; como critério de exclusão, estabeleceu-se não incluir a emergência devido ao menor tempo de permanência dos pacientes nesse setor.

A coleta dos dados ocorreu no período de 28 de junho a 28 de novembro de 2017, com o total de 111 participantes. As visitas ocorreram três vezes por semana, em dias e horários alternados, e foram realizadas por uma única pesquisadora, nos turnos da manhã e da tarde. Todos os cenários eram visitados nos dias de coleta e identificavam-se os pacientes maiores de 18 anos. Após a obtenção do consentimento informado, realizou-se a abordagem clínica para avaliação dos pacientes e preenchimento do instrumento de observação do uso de contenção mecânica.

O instrumento utilizado foi desenvolvido por Evans et al. para registrar o uso de contenção mecânica em idosos que residiam em ILPIs nos Estados Unidos ${ }^{17}$.Para utilização desse instrumento, solicitou-se autorização. Após o consentimento, realizaram-se adequações para aplicabilidade no cenário hospitalar, ou seja, mudaram-se as palavras associadas ao cenário de ILPI por Hospital.

Com esse instrumento, foi possível obterem-se informações de identificação de cada participante, se estava contido e qual tipo de contenção utilizado, data, gênero, idade e unidade de internação. Em seguida foram coletados os dados das variáveis: data de admissão, diagnóstico médico, motivos e tempo do uso de contenção mecânica, uso de dispositivos invasivos, presença e estadiamento de LP. As LPs foram identificadas durante a avaliação clínica. A equipe e/ou acompanhantes eram questionados sobre a presença de lesões e a pesquisadora avaliava tipo, local e estadiamento. Para complementar a observação direta, houve consulta ao prontuário dos pacientes.

A partir dos dados coletados, construiu-se um banco de dados no Microsoft Excel 2010 para análise pelo Statistical Package for the Social Science - SPSS versão 22.0. Para caracterização da amostra na análise descritiva do comportamento das variáveis, os dados foram sintetizados 
por meio do cálculo de estatísticas descritivas, gráficos, distribuições de frequências simples e tabelas cruzadas.

$\mathrm{Na}$ análise inferencial, as proporções de interesse também foram estimadas por intervalo de confiança (IC) para proporções. A significância da associação entre duas variáveis qualitativas foi investigada pelo teste qui-quadrado e, quando esse se mostrou inconclusivo, foi apropriado pelo teste exato de Fisher. A medida que expressou o risco foi a razão de chances [odds ratio $(\mathrm{OR})$ ], a qual avaliou a relação entre a chance de um indivíduo contido apresentar LP comparada à do indivíduo não contido, com IC maior do que 1.

O estudo foi aprovado pelo Comitê de Ética em Pesquisa do Hospital Universitário Antônio Pedro da Universidade Federal Fluminense sob o parecer 2.172.275.

\section{RESULTADOS}

Com relação ao gênero, no grupo de não contidos houve predomínio de pacientes do gênero feminino $(61,1 \%)$ e, entre os pacientes contidos, houve predomínio do gênero masculino (64,9\%). Quanto à distribuição de idades, observou-se concentração maior de pacientes com menos de 58 anos $(44,4 \%)$ no grupo de não contidos, enquanto entre os contidos a maioria tinha mais de 68 anos $(49,20 \%)$.

Dos 111 pacientes observados, encontraram-se $57 \mathrm{em}$ contenção mecânica, com prevalência estimada em 51,4\%. O tipo de contenção mecânica mais prevalente foi o uso de grades laterais no leito (100\%), sendo que em 70,2\% dos pacientes somente a grade era utilizada e $29,8 \%$ dos pacientes também estavam com os pulsos contidos. A justificativa para a contenção mais comum foi o risco de quedas (100\%) seguida do uso de dispositivos invasivos (57,9\%).

A ocorrência global de LP foi de 25,2\% (28 pacientes). No grupo de pacientes não contidos, a ocorrência de LP foi de 5,6\% (três pacientes), e no grupo de pacientes contidos a ocorrência de foi de 43,9\% (25 pacientes). Evidenciou-se associação entre o uso de contenção mecânica e a presença de LP. A OR de um paciente contido apresentar LP foi 13,3 vezes maior do que a de um paciente não contido, com IC significativo (3,7-47,6). A Fig. 1 mostra a ocorrência de LP nos dois grupos e na amostra global.

As LPs ocorreram principalmente na região sacra e se caracterizavam no estágio 2. A distribuição por localização e estágio da lesão pode ser vista nas Figs. 2 e 3, respectivamente.

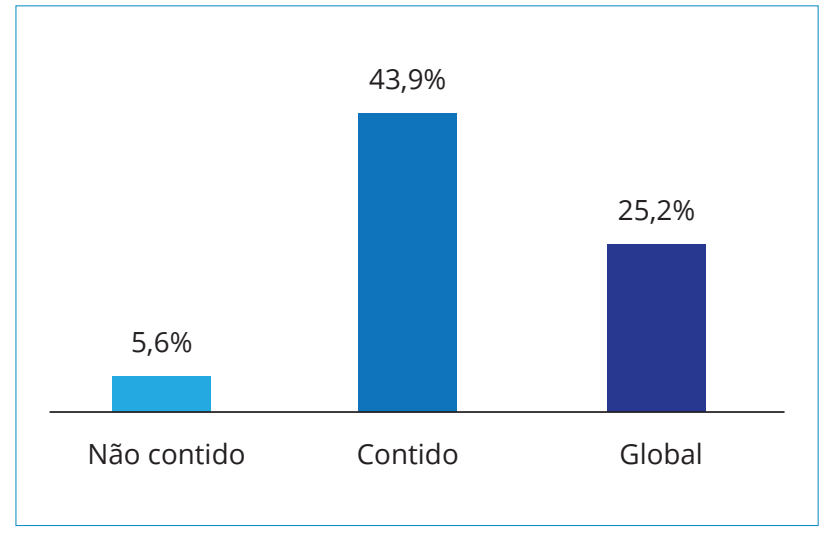

Figura 1. Ocorrência de lesões por pressão na amostra do estudo.

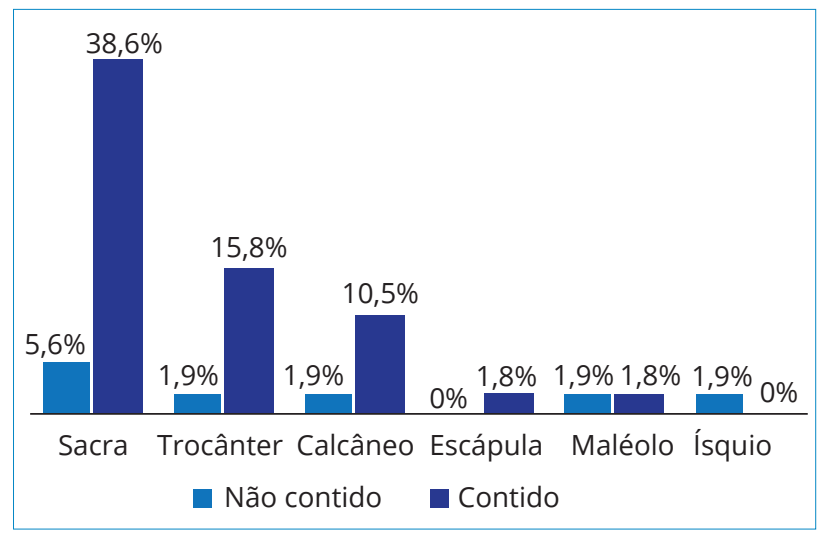

Figura 2. Ocorrência de lesão por pressão por localização anatômica da lesão.

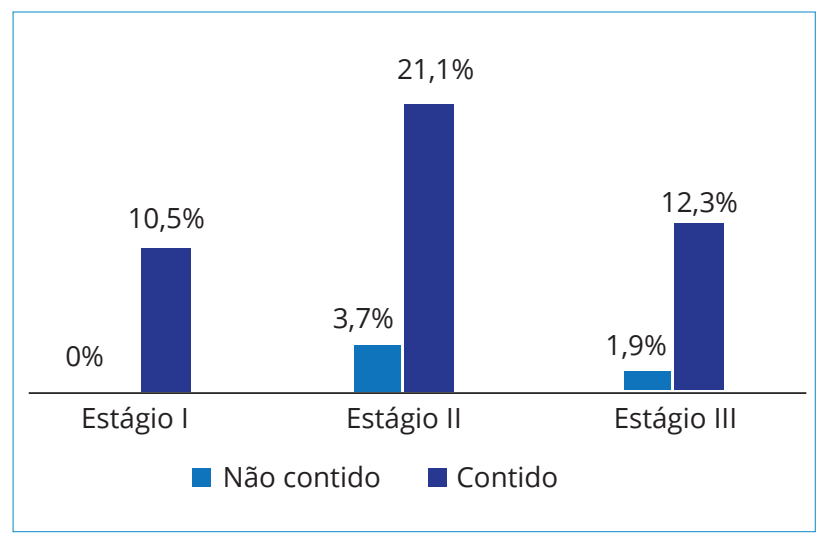

Figura 3. Ocorrência de lesão por pressão por estágio da lesão.

\section{DISCUSSÃO}

O principal dado desse estudo foi a associação entre a ocorrência de LP e a prática de contenção mecânica. A importância desse dado é demonstrada com a estimativa de 43,9\% de ocorrência de LP em pacientes contidos, totalizando 25 pacientes, enquanto no grupo de não contidos a ocorrência foi de apenas 5,6\%, ou seja, três pacientes. Verificou-se que as chances de pacientes contidos apresentarem LP 
foi 13 vezes maior em pacientes com contenção mecânica do que naqueles não contidos. Outros estudos também revelaram correlação entre o uso de contenção mecânica e o desfecho de LP, entretanto não utilizaram medidas de comparação em relação a pacientes não contidos. ${ }^{3,6}$

A incidência de LP é considerada importante indicador de qualidade assistencial em enfermagem e deve ser analisada quanto à sua distribuição, quais os pacientes mais vulneráveis e o local em que são mais frequentes ${ }^{8}$. Instrumentos de avaliação de risco para a integridade da pele foram validados, como as escalas de Norton, Gosnell, Waterlow e Braden, com a finalidade de nortear medidas para prevenção de $\mathrm{LP}^{16}$. Entretanto, nesses instrumentos de avaliação utilizados pela equipe de enfermagem, a contenção mecânica não é considerada fator predisponente. Estima-se que medidas preventivas possibilitam reduzir a incidência de LP em $50 \%$ e, dentre essas, inclui-se a redução do uso de contenção mecânica ${ }^{18}$.

Visando à prevenção e controle sistemático de danos que possam ser causados à clientela durante a assistência de enfermagem, a Resolução COFEN No 427 de 2012 recomenda o emprego da contenção mecânica somente quando esse for o único meio disponível para prevenir dano imediato ou iminente ao paciente ou aos demais, preferencialmente a partir de protocolos da instituição que determinem sua indicação, excetuando-se as situações de urgência e emergência ${ }^{19}$.

A contenção mecânica deve ser aplicada sob a supervisão direta do enfermeiro e, durante seu uso, a equipe de enfermagem deve proceder à inspeção da pele e da circulação nos locais e membros do paciente regularmente, bem como ao monitoramento clínico do nível de consciência, de dados vitais, em período não superior a uma hora. Pacientes sob sedação, sonolentos ou com algum problema clínico, bem como idosos, crianças e adolescentes, devem ser monitorados com maior rigor. $\mathrm{O}$ tempo de uso das contenções deve ser o estritamente necessário, e devem ser registradas em prontuário as indicações para emprego e a duração de seu uso, a ocorrência de eventos adversos, assim como os detalhes relativos ao monitoramento clínico ${ }^{19}$.

Portanto, a partir dos dados da pesquisa, sugere-se que pessoas com contenção mecânica tenham maiores chances para desenvolvimento de lesões por pressão, devendo ser instituídas medidas preventivas para manutenção da integridade da pele nesses pacientes, instituindo-se rigoroso monitoramento e avaliação dos índices de LP, considerados importantes indicadores de qualidade da assistência de enfermagem ${ }^{8}$.

Com relação à topografia, a região mais acometida foi a sacra, seguida da trocanteriana e do calcâneo. Os dados corroboram os resultados de outros estudos, nos quais houve predomínio das lesões na mesma topografia por serem regiões de apoio quando o paciente está em posição supina ou lateral ${ }^{8-10}$.

Os pacientes contidos apresentaram maior frequência de lesões nos estágios 2 (21,1\%) e 3 (12,3\%). Embora não seja possível estabelecer uma relação causal, já que em geral as LPs decorrem de diversos fatores associados, os pacientes com contenção mecânica muitas vezes têm mobilidade prejudicada, e observa-se que essa contenção reduz ainda mais a movimentação ${ }^{6,22}$, devendo seu uso ser descontinuado logo que possível. $\mathrm{O}$ tratamento de lesões nesses estágios implica em uso de maior quantidade de produtos, aumentando os custos da assistência ${ }^{24}$.

$\mathrm{O}$ uso de contenção prevaleceu no gênero masculino, sendo o mesmo achado verificado em outros estudos ${ }^{3,21}$. Quanto à idade, houve maior proporção de idosos contidos. Observa-se que esse segmento é mais exposto à contenção mecânica e, associada à restrição de movimentos pelo seu uso e a outros fatores relacionados ao envelhecimento, como maior fragilidade cutânea ${ }^{7}$, incontinência urinária e fecal ${ }^{8,22}$, aumentam as chances do desenvolvimento de LPs durante a internação.

Neste estudo, a razão citada pela equipe para o uso de contenção foi o risco de queda seguido do uso de dispositivos invasivos. Um estudo de caso-controle que relacionou o risco de queda com o perfil dos pacientes e o uso de medicação identificou que a queda do leito nos hospitais tem etiologia multifatorial, como problemas de marcha ou falta de força nos membros inferiores, frequência das eliminações fisiológicas, incontinência urinária e confusão, assim como o uso de medicação antipsicótica ou sedativa ${ }^{20}$.

$\mathrm{O}$ estudo apresentou como limitações o fato de a pesquisa ter sido realizada em apenas um hospital, nos turnos da manhã e da tarde e por uma única pesquisadora. Esta pesquisa, por ser transversal, não permitiu precisar o momento da ocorrência da LP no uso de contenção. Há necessidade de se aprofundar outros fatores que contribuem para a injúria tecidual e a gravidade dos pacientes que podem ser confundidores. Também não foi possível afirmar que há relação causal entre os resultados encontrados e o uso de contenção mecânica, mas sugere-se a realização de um 
desenho longitudinal, apesar das questões éticas limitantes, pois na medida em que há pacientes contidos a longo prazo e evoluindo com LP, a pesquisa deveria ser interrompida. Porém, destaca-se que foi possível identificar associação entre os fatores pesquisados que merecem outros estudos, como também a divulgação ampla sobre o tema.

\section{CONCLUSÃO}

Conclui-se que os pacientes em contenção mecânica têm maior chance de desenvolver LP, sobretudo aqueles idosos que estão mais predispostos devido ao déficit cognitivo e dificuldade motora, razões que convergem ao surgimento de LP, formulando um círculo vicioso que causa danos aos pacientes e comprometem a qualidade do cuidado de enfermagem.

No aspecto gerencial institucional, estratégias organizacionais e ambientais podem conduzir à redução do uso de contenção e, consequentemente, das taxas de queda. Recomendam-se abordagens para melhora cognitiva e motora do paciente, como mobilização e reposicionamento, higiene e cuidados diários com a pele e lesões. Contudo, é fundamental o desenvolvimento de ações educativas para prevenção de LP e o cumprimento dos procedimentos normatizados para uso da contenção mecânica.

\section{CONTRIBUIÇÃO DOS AUTORES}

\section{Conceitualização, Souza LMS, Santana RF e Menezes} AK; Metodologia, Santana RF e Rembold SM; Investigação, Souza LMS; Redação - Primeira versão, Souza LMS e Santana RF; Redação - Revisão \& Edição, Santana RF, Souza MV de, e Rembold SM; Aquisição de Financiamento, Santana RF e Souza LMS; Recursos, Santana RF e Souza LMS; Supervisão, Santana RF.

\section{FINANCIAMENTO}

Fundação Carlos Chagas Filho de Amparo à Pesquisa do Estado do Rio de Janeiro [https://doi. org/10.12039/501100004586]

Grant No.: 200.990/2017.

\section{REFERÊNCIAS}

1. Krüger C, Mayer $H$, Haastert B, Meyer G. Use of physical restraints in acute hospitals in Germany: a multi-centre cross-sectional study. Int J Nurs Stud. 2013;50(12):15991606. https://doi.org/10.1016/j.jinurstu.2013.05.005

2. Estêvez-Guerra G], Fariña-López E, Núñez-González E, Gandoy-Crego M, Calvo-Francés F, Capezuti EA. The use of physical restraints in long-term care in Spain: a multi center cross-sectional study. BMC Geriatrics. 2017;17(1):29. https://doi.org/10.1186/s12877-017-0421-8

3. Raguan B, Wolfovitz E, Gil E. Use of physical restraints in a general hospital: a cross-sectional observational study. Isr Med Assoc J. 2015;17(10):633-8.

4. Steinert $T$, Lepping $P$, Bernhardsgrütter R, Conca A, Hatling T, Janssen $W$, et al. Incidence of seclusion and restraint in psychiatric hospitals: a literature review and survey of international trends. Soc Psychiatry Psychiatr Epidemiol. 2010;45(9):889-97. https:// doi.org/10.1007/s00127-009-0132-3

5. Berzlanovich AM, Shöpfer J, Keil W. Deaths due to physical restraint. Deutsch Äztebl Int. 2012;109(3):27-32. https://doi. org/10.3238/arztebl.2012.0027

6. Bleijlevens MH, Wagner LM, Capezuti E, Hamers JP. Physical rRestraints: consensus of a research definition using a modified Delphi technique. J Am Geriatr Soc. 2016;64(11):2307-10. https://doi.org/10.1111/jgs.14435

7. Oearsakul B, Sirapo-Ngam Y, Strumpf NE, Malathum P. Physical restraint use among hospitalized elderly thais. Pacific Rim Int J Nurs Res. 2011;15(2):125-36.

8. Moraes JT, Borges EL, Lisboa CR, Cordeiro DCO, Rosa EG, Rocha NA. Conceito e classificação de lesão por pressão: atualização do National Pressure Ulcer Advisory Panel. Enferm Cent O Min. 2016;6(2):2292-2306. https://doi. org/10.19175/recom.v6i2.1423

9. Sanders LSDC, Pinto FJM. Ocorrência de úlcera por pressão em pacientes internados em um hospital público de Fortaleza-CE. Reme - Rev Min Enferm. 2012;16(2):166-70.

10. Borghardt AT, Prado TND, Bicudo SDS, Castro DSD, Bringuente MEO. Pressure ulcers in critically ill patients: incidence and associated factors. Rev Bras Enferm. 2016;69(3):460-7. https://doi.org/10.1590/0034$7167.2016690307 i$

11. He M, Tang A, Ge X, Zheng J. Pressure ulcers in the intensive care unit: an analysis of skin barrier risk factors. Adv Skin Wound Care. 2016;29(11):493-8. https://doi.org/10.1097/01. ASW.0000494779.66288.c9 
12. Arenas EB, Castañeda MDCP, Jiménez GP, Jiménez $P H$, Rodríguez JAR, Zárate MPP. Prevalencia de úlceras por presión en un hospital de tercer nivel, en México DF. Gerokomos. 2016;27(4):176-81.

13. Ricci JA, Bayer LR, Orgill DP. Evidence-based: the evaluation and treatment of pressure injuries. Plast Reconstr Surg. 2017;139(1):275e-86e. https://doi.org/10.1097/ PRS.0000000000002850

14. Greenhalgh DG. Management of the skin and soft tissue in the geriatric surgical patient. Surg Clin North Am.2015;95(1):10314. https://doi.org/10.1016/j.suc.2014.09.008

15. Belén FT. Formación y prevención en úlceras por presión: prevalencia en el Hospital General de Elche. Gerokomos. 2016;27(1):33-7.

16. Wechi JS, Amante LN, Salum NC, Matos E, Martins T. Escala de Braden: instrumento norteador para prevenção de úlcera por pressão. ESTIMA, Braz J Enterostomal Ther. 2017;15(3):14551. https://doi.org/10.5327/Z1806-3144201700030005

17. Evans LK, Strumpf NE, Allen-Taylor SL, Capezuti E, Maislin $\mathrm{G}$, Jacobsen B. A clinical trial to reduce restraints in nursing homes. J Am Geriatr Soc. 1997;45(6):675-81. https://doi. org/10.1111/j.1532-5415.1997.tb01469.x

18. Araújo TM, Araújo MFM, Caetano JÁ. O uso da escala de Braden e fotografias na avaliação do risco para úlceras por pressão. Rev Esc Enferm USP. 2012;46(4):858-64. https:// doi.org/10.1590/S0080-62342012000400011.
19. Conselho Federal de Enfermagem. Resolução COFEN Nº 427 de 07 de maio de 2012 [Internet]. Brasília, DF; 2012 [citado 14 Jan 2019]. Disponível em: http://www.cofen.gov. br/resoluo-cofen-n-4272012_9146.html

20. Krauss MJ, Evanoff B, Hitcho E, Ngugi KE, Dunagan WC, Fischer I, et al. A case-control study of patient, medication, and care-related risk factors for inpatient falls. J Gen Intern Med. 2005;20(2):116-22. https://doi.org/10.1111/j.15251497.2005.40171.x

21. Snaches B, Contrin LM, Beccaria LM, Frutuoso IS, Silveira AMR, Werneck AL. Adesão da enfermagem ao protocolo de lesão por pressão em unidade de terapia intensiva. Arch Health Sci. 2018;25(3):27-31. https://doi.org/10.17696/23183691.25.3.2018.1058

22. Hofmann H, Hahn S. Characteristics of nursing home residents and physical restraint: a systematic literature review. J Clin Nurs. 2013;23:3012-24. https://doi. org/10.1111/jocn.12384

23. Ministério da Saúde (BR). Portaria No 529 de 01 de abril de 2013 [Internet]. Brasília, DF; 2013. [citado 14 Jan 2019]. Disponível em: http://bvsms.saude.gov.br/bvs/saudelegis/ gm/2013/prt0529_01_04_2013.html

24. Silva DRA, Bezerra SMG, Costa JP, Luz MHBA, Lopes VCA, Nogueira LT. Curativos de lesões por pressão em pacientes críticos: análise de custos. Rev Esc Enferm USP. 2019;51:e03231. http://dx.doi.org/10.1590/s1980$220 \times 2016014803231$ 